\title{
PAOLO MANTEGAZZA: OLTRE LA SCIENZA
}

\author{
VITTORINO ANDREOLI (*)
}

SuNTO. - Anche uno scienziato è un uomo e non è giusto ridurlo a ciò che egli ha realizzato nei laboratori di ricerca. L'esistenza di un uomo è molto più ricca, è fatta di relazioni e di sentimenti e queste dimensioni incidono persino sulla razionalità, su cui si fonda la metodologia scientifica. Ho voluto per questo, dopo che molti hanno raccontato il Mantegazza scienziato, declinare la sua figura di uomo. Ed è così che è uscito il ritratto di un grande uomo, di un esempio da non dimenticare e di un ottimo farmacologo.

$$
* * *
$$

ABSTRACT. - Even a scientist is a man, and to reduce him down to what he has attained in research labs is not fair. A man's existence is much richer, composed of relationships and feelings and these relationships have an effect even on the rationality of which the scientific methodology is based. For this reason, after many have described the scientist Mantegazza, I wanted to illustrate his personal characteristics. And it is so that a portrait of a great man transpired, an example not to be forgotten, a great professor of pharmacology.

\section{PREMESSA STORICA}

Parlerò di Paolo Mantegazza, ma non del Mantegazza scienziato, pur avendolo conosciuto nell'Istituto di Farmacologia dell'Università Statale di Milano che io stesso ho frequentato dal ' 67 sino al '74. Un grande e prestigioso Istituto.

Il prof. Mantegazza nel '70 aveva vinto la cattedra a Milano.

Io non volevo diventare farmacologo, - ho salvato i farmacologi dall'avermi tra le loro schiere - quanto piuttosto di occuparmi di matti.

Sono diventato psichiatra, del resto l'Istituto di farmacologia di

${ }^{(*)}$ Psichiatra e scrittore, Istituto Lombardo Accademia di Scienze e Lettere, Milano, Italia, New York Academy of Sciences, USA.

E-mail: andreoli.vittorino@gmail.com 
Milano ha partorito uomini che sono poi giunti in diverse specialità cliniche e ciò è da leggere come un segnale della grande potenzialità di quell'Istituto.

Mi occupavo di comportamento dell'uomo e frequentavo i laboratori per studiare il cervello che era visto, giustamente, come l'organo del comportamento.

Provenivo dall'Università di Padova, dove lavoravo con Massimo Aloisi che si occupava di patologia muscolare, e pertanto, volendomi dedicare alla sofferenza mentale, presi coscienza che a Padova non potevo dare soluzione a questo mio desiderio.

Lo trovai invece all'Istituto di Farmacologia, diretto dal professor Emilio Trabucchi.

Non ho mai lavorato con il professor Mantegazza, però ho accettato volentieri di esprimere delle considerazioni su di lui, innanzitutto perché lo osservato all'interno di questo teatro farmacologico, ricco di scienza e di umanità. Io ho vissuto in quel periodo in una stanza dell'Istituto, e una stanza l'aveva anche il Direttore.

\section{UN VECCHIO PRINCIPIO}

Ha dominato nella Scienza un principio che si è imposto fino a poco tempo fa: sugli scienziati non c'è niente da dire: bisogna solo raccontare cosa hanno scoperto.

Ovviamente, quando si dice che quello scienziato è stato insignito del premio Nobel, si può mettere già un punto, perché si è implicitamente detto tutto: vuol dire che ha ottenuto un riconoscimento internazionale per i suoi contributi importanti dati alla scienza, nel campo specifico della sua attività.

Devo ammettere che l'idea di ridurre la valutazione di uno scienziato a ciò che ha fatto, dal mio punto di vista, ma non solo mio, è fortemente limitativo. A me pare che si debba sempre tenere presente che uno scienziato è comunque un uomo, e che quindi sia giusto conoscere anche la sua personalità, il suo ampio raggio di esperienza e di esistenza.

E questa convinzione trova la conferma anche nel caso di Mantegazza, un uomo molto ricco sul piano affettivo e su quello degli interessi (basti pensare alla sua conoscenza profonda sugli uccelli).

Attratto dall'uomo. e dunque da una pluralità di parametri con cui valutarlo, mi sono chiesto se la mia esperienza diretta, fatta in tanti 
anni di osservazione, e avendo parlato molte volte con lui anche successivamente, quando ritornavo già psichiatra, in Istituto, era tale da consentirmi una cognizione sufficientemente profonda da ricordarlo oggi.

$\mathrm{E}$ ho concluso che qualcosa potevo dire.

Era un farmacologo, uno scienziato dunque, ma attentissimo agli aspetti della clinica, e quando mi vedeva, voleva sentire parlare della neurologia di cui ero specialista, della psichiatria che praticavo e soprattutto del malato.

Un interesse che avevo trovato anche nel professor Trabucchi, il suo maestro, il quale nello studio aveva disposto una stanzetta con un lettino per visitare i pazienti.

Il prof. Mantegazza con me parlava dei giovani, dell'esordio della schizofrenia e di tutte le forme mentali, mostrando una conoscenza che non era certo comune.

Insomma, sono convinto che degli scienziati sia giusto che si osservi anche qual è la loro dimensione umana. Credo che se uno volesse scrivere una biografia di questo personaggio, dovrebbe partire dalla scienza, ma dedicarsi anche a ciò che non è scientifico, perché anche questa è una solida modalità per capirlo.

Ci sono esempi di grandi scienziati - grandi uomini, ma anche di molti che non meritano uno speciale rispetto sul piano della loro umanità.

Albert Einstein è stato di certo uno degli scienziati più celebrati della storia, ha avuto il riconoscimento del Nobel Prize e non per il suo contributo principale, la relatività, che rientrava più nella matematica esclusa da questa competizione. Grande scienziato dunque, ma pessimo marito e un padre orrendo. Dalla prima moglie aveva avuto un figlio che soffriva di schizofrenia, e che non ha mai voluto vedere.

Charles Darwin era una personalità meravigliosa, un padre e un marito affettuoso; nonostante il suo lungo viaggio alla ricerca delle origini delle specie, preferiva stare a casa, cercando di realizzare un'esistenza familiare.

Queste diverse combinazioni valgono anche per le arti. Nella pittura. Michelangelo, il nostro più celebrato artista, era tirchio, avarissimo. Non amava le relazioni, era un misantropo. Non si sposò mai, visse sempre molto poveramente, con una cassa piena di denari nascosti.

Caravaggio ha commesso addirittura un omicidio.

Accettando l'invito a parlare di Paolo Mantegazza, ho dunque infranto l'idea che di uno scienziato si debba parlare solo dell'aspetto 
scientifico; e devo dire che sono particolarmente lieto di farlo perché a me sembra un buon esempio di uomo.

Lo dico qui, di fronte a molti scienziati, ricordando che lo scienziato universitario ha anche il compito della docenza, (da docere, insegnare), e questo vuol dire avere relazioni con gli studenti, e possedere caratteristiche comunicative, umane, e quindi mi sembra che il prof Mantegazza sia un esempio per sostenere la mia visione e cioè che per prima cosa di un uomo bisogna vedere quanto è uomo, poi quanto è professionista o scienziato.

Anche nella mia disciplina ci sono grandi psichiatri che però sono dei piccolissimi uomini. E forse si può capovolgere il binomio, aggiungendo che ci sono anche uomini di grande peso, di grande esempio, pur se non possono essere menzionati per delle grandi scoperte.

Avendo ascoltato i risultati scientifici ottenuti dallo scienziato Mantegazza, non è questo il caso e io ne sono orgoglioso perché le sue ricerche sono per la maggior parte realizzate in quell'Istituto in cui io ho lavorato per prepararmi ad andare in mezzo al dolore mentale.

\section{L'UOMO E LO SCIENZIATO}

Io non avrei potuto dire niente della sua scienza e delle sue ricerche, però forse posso tratteggiare, sulla base delle mie osservazioni il Mantegazza-uomo. Era questo l'aspetto che valutavo, quando parlavamo insieme; ma ho anche voluto parlare con i suoi allievi, con chi lo conosceva bene, anche per i suoi interessi umani. Ho inoltre raccolto le esperienze che in qualche modo mi potevano dare delle indicazioni, naturalmente escludendo sempre la famiglia, perché fare una biografia significa rispettare il secretum: c'è un privato che appartiene solo all'ambito familiare e lì deve restare.

Io nutro grande apprezzamento per un filosofo, Arthur Schopenhauer, il quale scrisse un libro - in realtà era una serie di articoli - che venne pubblicato postumo con il titolo Insegnare a vivere. Il libro contiene suggerimenti per vivere bene. Sosteneva che dell'uomo bisogna chiedersi chi è, prima domanda, che cosa ha, seconda domanda, e che cosa rappresenta per gli altri e per la società.

La scienza in sostanza racconta che cosa Mantegazza ha fatto, che cosa ha scoperto, quanto io dirò appartiene al chi è (chi è stato), tenendo sempre presente quella presunzione necessaria a chi fa il mio 
mestiere, quella di saper focalizzare la interiorità, la personalità, la mente.

Elencherò gli elementi che secondo me definiscono un uomo, il suo trovarsi dentro l'umanesimo, e posso anticipare che molti di questi elementi appartengano a questo straordinario personaggio, a Paolo Mantegazza; e se lo è, allora ne deriva che ha fatto bene l'Accademia di Scienze Lettere e Arti di Milano a ricordarlo, poiché rimane un esempio da proporre. C'è una grande carenza di esempi, in questo momento storico, anche all'Università e nella Clinica di cui mi occupo, e averne è molto importante.

\section{Questo È UN UOMO}

La caratteristica principale dell'uomo è il dolore, il dolore esistenziale. Quel dolore che non ha un organo, un riferimento, prende proprio tutta l'esistenza, tutta la personalità. Chi si occupa di follia conosce soprattutto questa espressione del dolore, il dolore esistenziale.

Io ho visto Mantegazza nel dolore esistenziale, ero in Istituto quel gennaio del '72. E se ne deve parlare poiché dobbiamo trasformare in gioia ogni accadimento umano, altrimenti ci carichiamo soltanto del dolore. mistero.

Il dolore va visto dentro l'avventura esistenziale, dentro il suo

Io ricordo bene la mia cara nonna Virginia. Qualche volta la scoprii che piangeva. Le chiedevo: ma che cos'hai nonna? Non ti preoccupare, mi diceva, piango di gioia. La gioia non è felicità che appartiene al singolo, la gioia è corale, riguarda il Noi, e diventa appartenenza.

Io l'ho visto in quel periodo. E mi chiedevo, poiché l'evento di cronaca era tra quelli mostruosi, perché mai venisse in Istituto, uscisse dalla cerchia familiare in cui viveva, e lo guardavo e lo osservavo.

Ho visto il volto del dolore, ma non era un dolore disperato; e forse molta parte delle cose che poi ha fatto gli sono servite perché quel dolore non arrivasse alla disperazione.

Mantegazza era molto religioso, anche se non ne dava trasparenza, non era una religiosità teatrale, ma intima, e la religione sa dare al dolore un significato. Un significato umano.

No, non l'ho visto mai disperato.

Continuava a dare l'esempio, a insegnare attraverso l'esempio. 
Aveva perduto due figlioli in un attimo. Li conoscevo, soprattutto il ragazzo. Un ragazzo meraviglioso. Per Mantegazza, era successo qualcosa di misterioso, e nel mistero, è difficile disperarsi, perché ti sfugge e allora ti devi sempre attaccare alla speranza, Soren Kierkegaard definisce la disperazione, la malattia mortale.

Il Mantegazza e la sua religiosità.

Sono andato a vedere tutte le onorificenze da lui ricevute. Sono molte. Una l'ha avuta dal cardinale Martini: gli è stata data la onorificenza papale - allora c'era papa Giovanni Paolo II -. Aveva una relazione con un sacerdote, un prete con cui forse parlava di Dio. Il prete e lo scienziato, il Rettore Magnifico, a parlare di Dio. Un argomento non scientifico ma capace di dare forza a vivere.

E quel dolore è proprio la cifra che caratterizza l'uomo; solo l'uomo prova il dolore esistenziale.

Lui l'ha provato, e quando si fermava con qualcuno, in quei giorni, spesso non diceva niente.

Dopo il dolore, la religiosità, che è il senso sacro del mondo.

In un'occasione in cui si parlava di credenti e di non credenti, lui disse che forse poteva anche avere dei dubbi, ma che era impossibile avere speranza senza immaginare un Dio a cui credere.

Una bella espressione: la speranza è in qualche modo una modalità per vincere il dolore, quel dolore, che si è depositato dentro di sé. E' una forza perché divenga ricordo.

Una delle qualità che Mantegazza aveva in maniera straordinaria, era il silenzio.

L'Istituto di farmacologia era un teatro vastissimo, in alcuni periodi eravamo in novanta persone e tutti parlavano; Mantegazza parlava sempre poco. Preferiva farlo nel suo Studio, dove però sapeva ascoltare a lungo, e i suoi allievi conoscevano che se la porta era chiusa non bisognava entrare, anche se la segretaria diceva che non c'era nessuno; se la porta era aperta si poteva, e lui ascoltava, ascoltava tutti.

Una volta sono entrato nello Studio, mi aveva invitato a parlare in Facoltà, e volevo capire che cosa si aspettasse da me. Abbiamo parlato di matti.

Sorrideva poco, ma faceva sorridere talvolta.

Io amo molto i clown, perché vogliono dare gioia sentendo la malinconia.

Ti offriva quel suo volto, quella sua serenità. Sapeva essere gioioso, nonostante avesse subito nella vita delle prove molto dure. 
Ho letto la relazione che ha tenuto quando ha terminato l'attività di rettore. $\grave{E}$ un documento che dovrebbe essere studiato profondamente. Non ha voluto parlare di quello che ha fatto, decisioni, opere complicate perché calate all'interno di fazioni. Sappiamo qual è la vita pratica. Volle parlare del silenzio, della bontà.

Ho a lungo indagato tra i suoi allievi, per cercare di capire il personaggio. Nessuno che mi abbia detto: ma sai l'ho visto una volta fuori di sé. Era sempre tranquillo, non un buonista, ma chi cercava di capirti, di comprendere le tue difficoltà. E c'erano gli assistenti più giovani che andavano - già lo si faceva con Trabucchi, - a ricordare ancora questa linea comune - a raccontargli delle loro fidanzate, andavano a parlare della loro vita privata, perché avevano bisogno di un consiglio.

Era anche un maestro. Del resto non si è maestri solo insegnando una tecnica di laboratorio fondamentale per la ricerca. Il maestro è uno che ha la caratteristica di ascoltare e che ama il silenzio: una combinazione molto rara, ed è fortemente umana.

Se l'umanesimo si fondasse meno sul chiacchierare, meno sull'estroversione e forse un po' più sul silenzio, e guardasse l'altro per capirlo e non per considerarlo un nemico da abbattere perché ti ostacola, la società andrebbe meglio.

Ho trovato conferma in molti, era un uomo che aveva un grande rigore morale, e io uso questa parola molto raramente.

Oggi faccio fatica a incontrare qualcuno dei personaggi importanti, che non mi dica: "Sa io ho due processi in corso". Se uno non ne ha nessuno, come nel mio caso, vuol dire che è proprio un nessuno. I processi in corso bisogna averli, perché è segno che hai nemici, e i nemici sono "tanto onore".

Lui non ne ha avuto: è stato nelle battaglie più drammatiche, è riuscito a fare le cose più incredibili, non solo quelle per gli uccelli (porre grandi voliere in Istituto), senza mai sbraitare. Al contempo un uomo di grande determinazione.

E' riuscito a cambiare l'Istituto di farmacologia che sembrava imperituro, e l'ha cambiato in un anno; ha mutato il volto dell'Università. Riusciva a realizzare, con la sua capacità da grande diplomatico e sempre nel rispetto dell'altro, tutto quanto gli pareva giusto e utile alla Università.

Una persona determinata che però ascoltava gli altri e riusciva a non litigare, a non baruffare, a non considerarsi mai in guerra.

Permettetemi di usarla anche se è una parola ormai cancellata dai 
dizionari della Lingua italiana: era un uomo onesto, questa è la mia percezione.

Certo nell'Istituto di farmacologia uomini onesti ce ne sono stati, e non posso dimenticare la figura del professor Emilio Trabucchi, che è stato un Maestro, anche di Mantegazza.

Ma voglio aggiungere un'altra dote che mi sembra importante e che fa parte delle caratteristiche dell'umanità, la lungimiranza.

$\mathrm{Ha}$ condotto ricerche che hanno intuito cose che sono state completate da altri, era capace di intravvedere che cosa sarebbe stato utile, e l'ha incominciato. Questa è lungimiranza.

Ciò che lo ha caratterizzato sempre, è stata la passione.

So che voi tutti siete molto attivi, fatte in modo di dare un po' di passione agli studenti che avete davanti nelle aule dell'Università, perché questa è la carenza più forte che oggi riscontriamo nel mondo giovanile: non c'è più passione, passione certo per cose che riguardano anche il sapere.

Ho ascoltato la segretaria che Mantegazza aveva negli ultimi anni. Ho saputo che usava i bigliettini per indicare ai suoi allievi qualche articolo da leggere, ma la cosa che mi ha più colpito è che chiedeva alla segretaria di fare delle fotocopie quasi sempre di fotografie, e di farle a colori. Ebbene io ne ho viste alcune, ed erano quasi sempre squisitamente umane. In una erano rappresentate due bambine di colore, certamente di una condizione esistenziale che noi occidentali chiamiamo povertà, sorridevano di fronte a degli uccelli.

Mentre leggeva articoli scientifici, raccoglieva delle espressioni umane che riguardavano, questa volta, le difficoltà, che girano nel mondo, ragazzi e bambini che soffrivano.

E una cosa bella e commuove rilevare che uno scienziato ha questa sensibilità umana per quelli che non hanno nulla.

E forse qui è bello inserire il richiamo al negozio di panetteria del padre, che è poi un luogo di creazione.

Voglio finire con le sue parole, con quello che lui ha letto quel giorno - eravamo nel 2001 - dopo che avevano parlato tutti: "Ora tocca a me il compito di dire due parole, anche se mi rendo conto che sono troppo emozionato per fare un discorso che abbia un minimo di senso. Quello che è stato detto di me, mi ha profondamente colpito. Credo di non meritare tante espressioni di stima e di affetto, e di una così numerosa presenza di colleghi che sono convenuti nell'aula magna."

Avete parlato troppo bene di me. La modestia. E parla di quella 
porta aperta, e parla del silenzio: "Ho sempre avuto l'abitudine, e i miei colleghi lo sanno, che ricevendo qualcuno nel mio studio, avevo l'abitudine di allargare il discorso al di là dell'argomento per il quale era venuto a parlare..."

Questa è l'origine del titolo che ho dato a questo mio intervento: Oltre la scienza. 